\title{
Self-Assembled Strap for Global Proteomics and Salivary Biomarker Discovery
}

Yi-Han Lin ${ }^{1}$, Rodrigo Vargas Eguez ${ }^{1}$, Manolito G. Torralba², Harinder Singh ${ }^{1}$, Pawel

Golusinski $^{3,4,5}$, Wojciech Golusinski ${ }^{4}$, Michal Masternak ${ }^{6}$, Karen E. Nelson ${ }^{1,2^{*}}$, Marcelo Freire ${ }^{2^{*}}$, Yanbao $\mathrm{Yu}^{1 *}$

1. J. Craig Venter Institute, 9605 Medical Center Drive, Rockville, MD 20850

2. J. Craig Venter Institute, 4120 Capricorn Lane, La Jolla, CA 92037

3. Department of Otolaryngology and Maxillofacial Surgery, University of Zielona Gora, Zielona Gora, Poland

4. Department of Head and Neck Surgery, Poznan University of Medical Sciences, Greater Poland Cancer Centre, Poznan, Poland

5. Department of Biology and Environmental Studies, Poznan University of Medical Sciences, Poland.

6. College of Medicine, Burnett School of Biomedical Sciences, University of Central Florida, Orlando, FL 32827

" Corresponding author: Yanbao Yu, email: yayu@jcvi.org, tel: (301) 795-7868; Marcelo Freire, email: mfreire@jcvi.org, tel: (858) 200-1846; Karen E. Nelson, email: Kenelson@jcvi.org, tel: (301) 795-7565.

\section{Supporting Information:}

Figure S-1. Pearson correlation analyses of log2-transformed protein intensities across the eight replicate experiments of the two filter types, commercial STrap and home-packed STrap.

Figure S-2. Representative Venn diagrams of the proteins and peptides identified in the first and second digestion of commercial STrap filter and consecutive or parallel tryptic digestion of home-packed STrap filter using HeLa lysate.

Figure S-3. Pearson correlation analyses of saliva samples for oral cancer biomarker discovery.

Supplementary Protocol. Protocol for self-assembled suspension trapping (STrap) filterbased digestion 
Table S-1 to S-4, attached separately.

Table S-1: Meta data of human subjects involved in this study.

Table S-2. Identification rate of commercial STrap filters and home-made STrap filters.

Table S-3. Label-free quantitation of OSCC subjects versus control subjects.

Table S-4. Significant proteins between OSCC and control subjects.

Supporting Information Figure S-1. Pearson correlation analyses of log2-transformed protein intensities across the eight replicate experiments of the two filter types, commercial STrap and home-packed STrap.

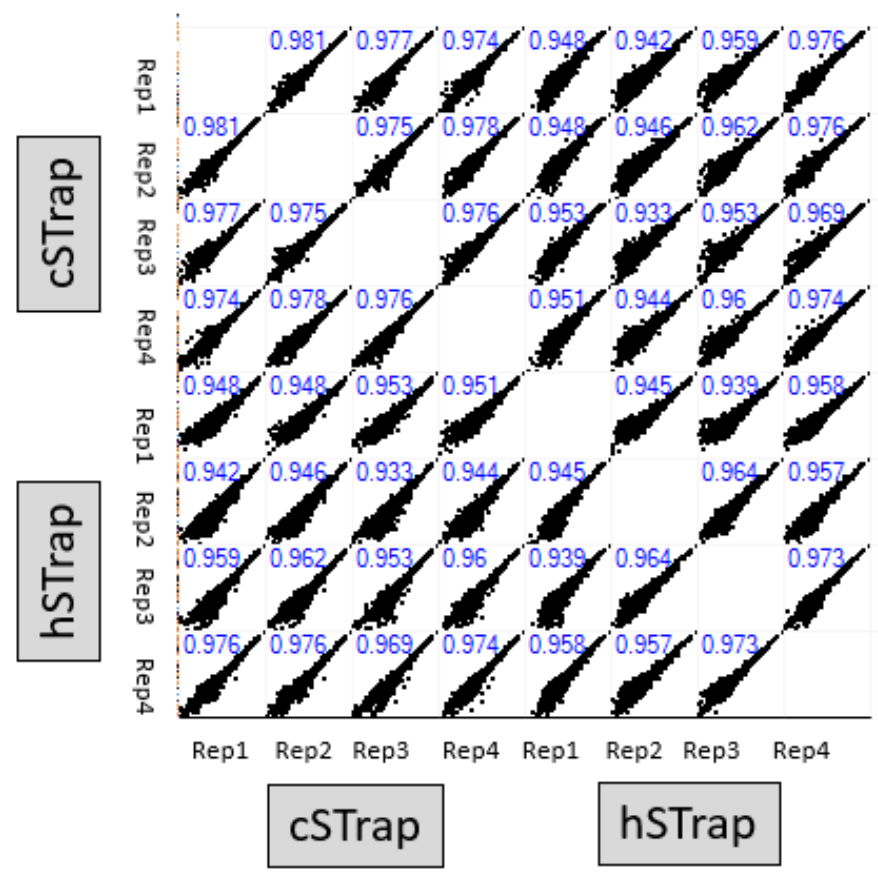


Supporting Information Figure S-2. (ㅅ) Representative Venn diagrams of the proteins and peptides identified in the first and second digestion of commercial STrap filter. For this test, a bacterial lysate of K. pneumonia was used (as described in PMID: 30114372). Quadruplicate tests were performed for each digestion. The consecutive digestion experiment was performed in three separate days using more than 10 STrap filters, and similar results were obtained from all experiments. Right panel shows the overlaps of two parallel tryptic digestion. (B) Consecutive or parallel tryptic digestion of home-packed STrap filter using HeLa lysate.

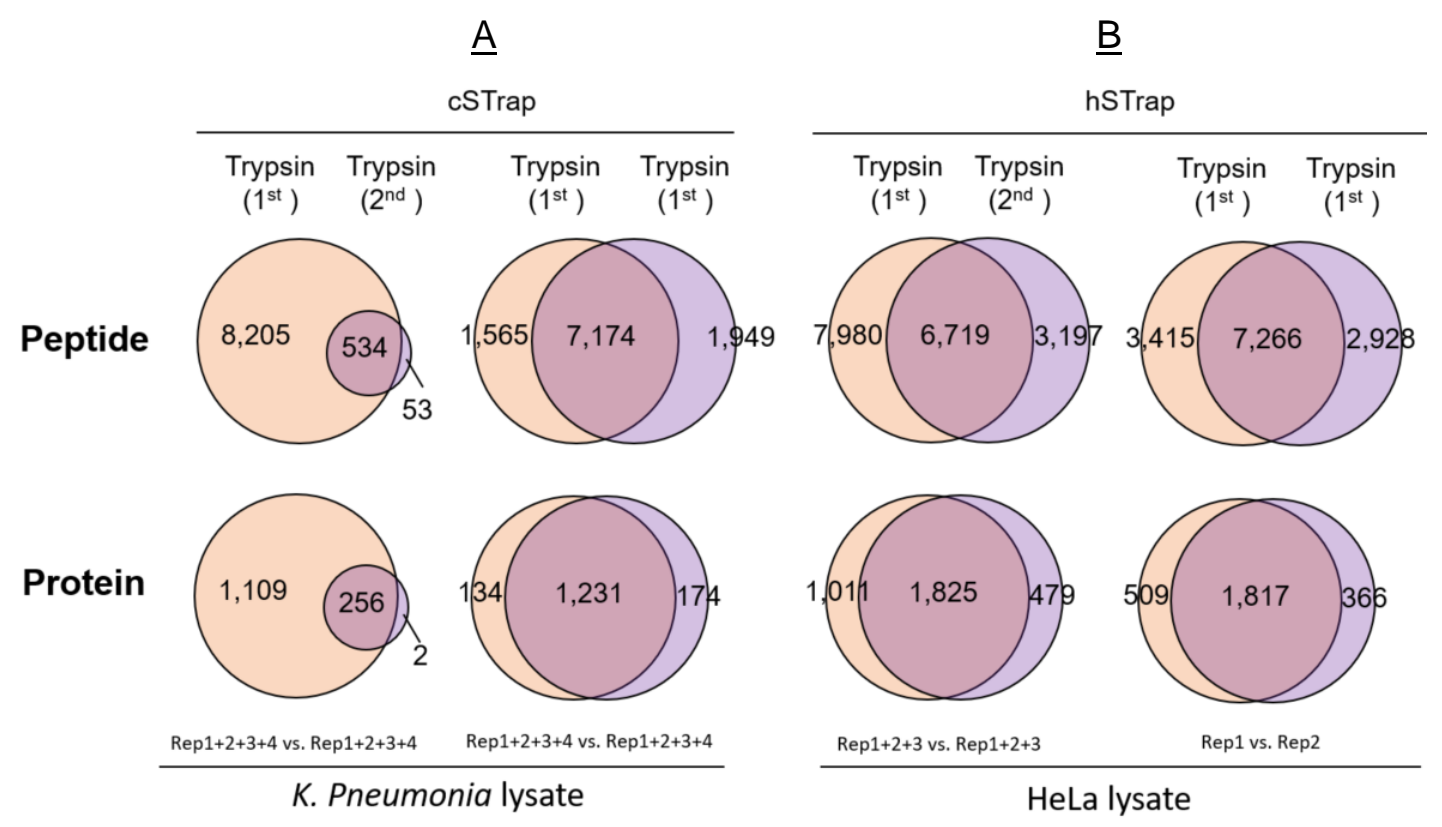


Supporting Information Figure S-3. Pearson correlation analyses of saliva samples for oral cancer biomarker discovery. As a note, no imputation (of missing values) or filtration (of low occurrence data) was performed before the analyses. ()ㅡ Correlations between different technical replicates (rep1 vs. rep2) of the same subject from the control group. The average and standard deviation values were derived from five experiments, as highlighted in red boxes. (B) Correlations between different technical replicates (rep1 vs. rep2) of the same subject from the cancer group. () Correlations between different subjects within or between the control and cancer group, respectively.
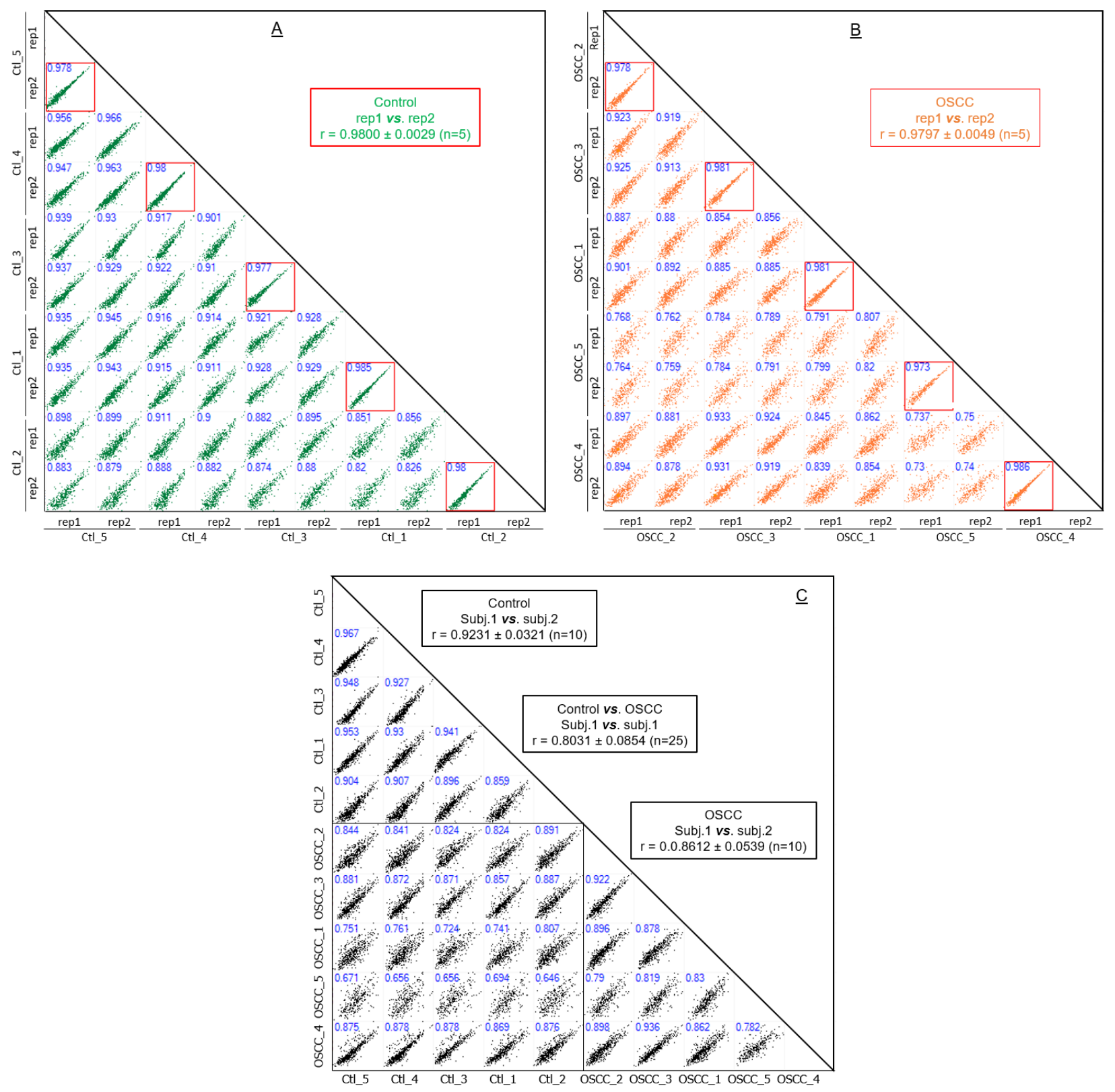


\section{Protocol for self-assembled suspension trapping (STrap) digestion}

\section{Materials and Reagents}

1) Whatman GF/F microfiber membrane circle or sheet (Cat. \#: 09-874-73, or 09-924-545; Fisher Scientific).

2) FASP filter devices.

Both Microcon (for instance, cat. \#: MRCF0R030, Millipore) and Vivacon (for instance, cat. \#: 14-558-344, Fisher Scientific) filters that are commonly found in proteomics laboratory can be used. Recycle used filters and detach/remove base with hand (or a wrench if difficulty is observed). Discard used membrane and sonicate the device component parts (Oring, base and tube, as shown on the right image) in a clean glass bottle

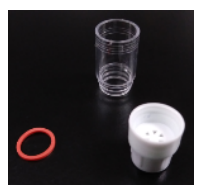
with HPLC water for 10 30 min. Air dry and store in a clean container for future use.

3) $5 / 16$ " hole punch.

Please select a vendor of hole punch on your own choice according to the price and quality. Please ensure the shape of hole punch is round circle, and the size is $\mathbf{5 / 1 6}$ " (or 10/32"). Since the glass fiber membrane is very soft, there is no special requirement for sharpness of hold punch. For this study, we purchased the "Economy Hole Punch - 9 Shapes, 2" Reach. 5/16" Round Hole" from Online River, LLC (https://onlineriver.com/economy-hole-punch-9-shapes-2-reach/).

4) Collection tubes (Cat. \#: 14-556-111, Fisher Scientific).

5) STrap buffers:

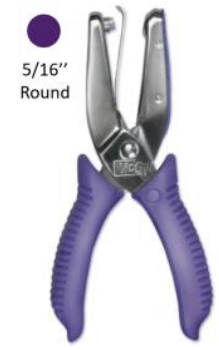

- Lysis buffer: 10\% SDS, 100 mM triethylammonium bicarbonate (TEAB), pH 7.55;

- Binding buffer: 90\% Methanol, 100 mM TEAB, pH 7.1;

- Neutralization buffer: $12 \%$ phosphoric acid in water;

- Digestion buffer: $50 \mathrm{mM}$ TEAM in water.

- Elution buffers: a) $50 \mathrm{mM}$ TEAB, b) $0.2 \%$ formic acid (FA), c) $0.2 \% \mathrm{FA} / 50 \% \mathrm{ACN}$.

6) STrap digestion reagents:

- Dithiothreitol, DTT

- Iodoacetamide, IAA

- Sequencing grade trypsin

\section{Methods}

\subsection{Filter packing and assembling}

1) The new membrane circle and the filter parts can be easily packed and assembled as illustrated in the diagrams below. One or two layers of membrane disc can be packed.
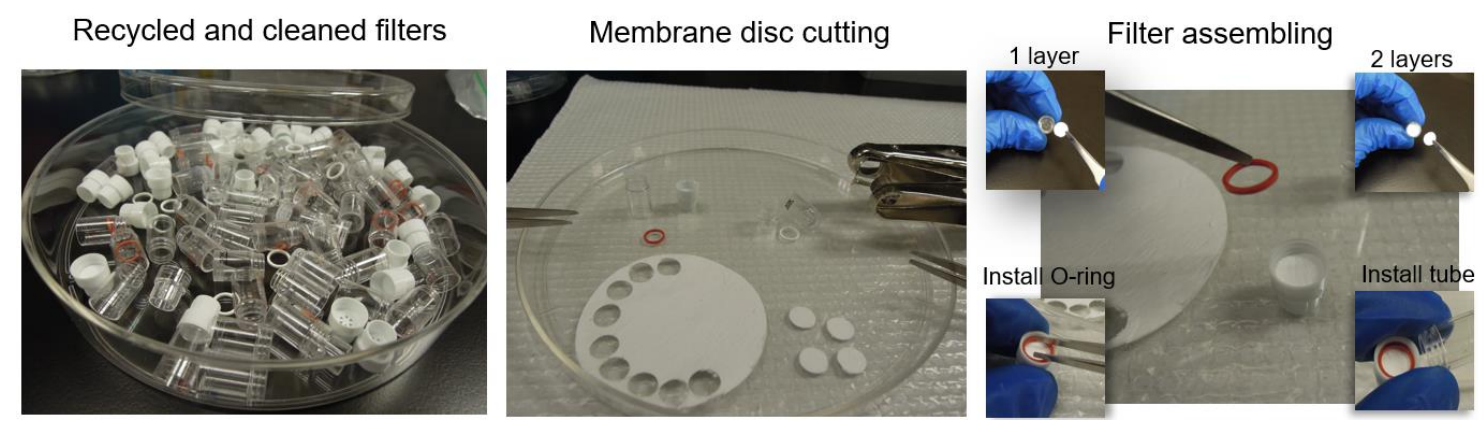
2) After assembling, transfer filter device to a collection tube, flush membrane with $400 \mu \mathrm{l}$ Binding buffer by centrifuging at 2,000 rpm for around $2 \mathrm{~min}$.

3) Take 10 50 $\mu$ g protein samples (e.g., whole cell or tissue lysate, body fluids), mix with equal volume of Lysis buffer, and boil at $95^{\circ} \mathrm{C}$ for $10 \mathrm{~min}$ with $20 \mathrm{mM}$ DTT.

4) Cool protein samples to room temperature, add IAA to a final concentration of $40 \mathrm{mM}$. Incubate samples in the dark for $30 \mathrm{~min}$.

5) Add $12 \%$ phosphoric acid to the proteins for a final concentration of $1.2 \%$, mix gently.

6) Add 6x Binding buffer to the proteins, incubate for 1 2 min with gentle mixing.

7) Load up to $400 \mu \mathrm{l}$ samples onto STrap filter device, spin at 2,000 rpm for 1 2 min, collect flow through and reload onto filter. Repeat this step three times.

Here, if the total sample volume is larger than $400 \mu$, split samples and do sequential loading.

8) After loading, discard flow through, and wash samples by flushing $400 \mu$ l Binding buffer through the filter. Repeat this step 1 2 times.

9) Transfer the filter device to a new collection tube. Add $150 \mu$ l Digestion buffer with proper amount of trypsin (Trypsin:Protein $=1: 50$ ), and incubate overnight at $37^{\circ} \mathrm{C}$. NO shaking is required.

10) To elute peptides, sequential elution with three Elution buffers. Pool elution and dry in SpeedVac.

11) Proceed to desalt samples using $\mathrm{C}_{18}$-based StageTip.

12) De-assemble the filter device (discard used membrane disc and collection tube), and recycle and clean the plastics for future use (back to Step 1). 